\title{
Autonomous FYDPS Neural Network-based Planner Agent for Health Care in Geriatric Residences
}

\author{
Juan F. de Paz, Yanira de Paz, Javier Bajo, Sara Rodríguez and Juan M. Corchado
}

Departamento Informática y Automática, Universidad de Salamanca

Plaza de la Merced s/n 37008, Salamanca, Spain

\{ fcofds, , yanira, jbajope, srg, corchado \}@usal.es

\begin{abstract}
This paper presents an autonomous intelligent agent developed for health care in geriatric residences. The paper focuses on the construction of an autonomous agent which incorporates a model of human thinking, such as reasoning based on past experiences. The work here presented focuses in the development of the CBP internal structure. The planning mechanism has been implemented by means of a novel FYDPS neural network. The system has been tested and this paper presents the results obtained.
\end{abstract}

\section{Introduction}

In this article we present a novel planning system based on the combination of neuronal networks with CBP (Case-based planning) systems [7]. Case-based planning allows us to retrieve past experiences when a new plan is created which lends the system a large capacity for learning and adaptation [7]. The neuronal networks proposed within this research framework are self-organised, based on Kohonen networks [10], but which present certain improvements (FYDPS neural Neural Network) [11]. These improvements allow the network to reach a solution much more rapidly. Furthermore, once a solution has been reached, it makes it possible to make new modifications taking restrictions into account (in this study, specifically time restrictions). The new planning mechanism is integrated within AGALZ (Autonomous aGent for monitoring ALZheimer patients) [5], [6], a planning agent that works in conjunction with complementary agents into a prototype multi-agent system (ALZ-MAS: ALZheimer Multi-Agent System). The results obtained are compared to those obtained with the previous geodesic planner used by AGALZ.

This work focuses in the development of deliberative agents using a case-based planning [1] architecture, as a way to implement adaptive systems to improve assistance and health care support for elderly and people with disabilities, in particular with Alzheimer's. Agents in this context must be able to respond to events, take the initiative according to their goals, communicate with other agents, interact with users, and make use of past experiences to find the best plans to achieve goals, so we propose the development of a deliberative agent that incorporates a CBP mechanism, specially designed for planning construction. CBP-BDI facilitates learning and 
adaptation, and therefore a greater degree of autonomy than that found in pure BDI (Believe, Desire, Intention) architecture [2].

During the last three decades the number of Europeans over 60 years old has risen by about $50 \%$. Today they represent more than $25 \%$ of the population and it is estimated that in 20 years this percentage will rise to one third of the population, meaning 100 millions of citizens [3]. This situation is not exclusive to Europe, since studies in other parts of the world show similar tendencies. The importance of developing new and more reliable ways to provide care and support to the elderly is underlined by this trend [3], and the creation of secure, unobtrusive and adaptable environments for monitoring and optimizing health care will become vital. Tomorrow's health care institutions will be equipped with intelligent systems capable of interacting with humans. Multi-agent systems and architectures based on intelligent devices have recently been explored as supervision systems for medical care for the elderly or Alzheimer patients, these intelligent systems aim to support them in all aspects of daily life, predicting potential hazardous situations and delivering physical and cognitive support.

In the next section the autonomous planner agent is presented. Then the new planning mechanism is described, finalizing with a case study and results and conclusions obtained after the implementation of a prototype into a real scenario.

\section{Autonomous Health Care Agent}

The CBP is based on the generation of plans from cases. The deliberative agents, proposed in the framework of this investigation, use this concept to gain autonomy and improve their guiding capabilities. The relationship between CBP systems and BDI agents can be established by implementing cases as beliefs, intentions and desires which lead to the resolution of the problem. In a CBP-BDI [5], [6] agent, each state is considered as a belief; the objective to be reached may also be a belief. The intentions are plans of actions that the agent has to carry out in order to achieve its objectives [8], so an intention is an ordered set of actions; each change from state to state is made after carrying out an action (the agent remembers the action carried out in the past, when it was in a specified state, and the subsequent result). A desire is any of the final states reached in the past (if the agent has to deal with a situation, which is similar to one in the past, it will try to achieve a similar result to the one previously obtained). Below, the guiding mechanism, used by the CBP-BDI agent, is presented: Let $E=\left\{e_{0}, \ldots, e_{n}\right\}$ the set of the possible rooms and places in the residence.

$$
a_{j}: \underset{e_{i}}{E} \rightarrow \underset{a_{j}\left(e_{i}\right)=e_{j}}{E}
$$

An Agent plan is the name given to a sequence of actions (1) that, from a current state $e_{0}$, defines the path of states through which the agent passes in order to offer to the nurse the better path. Below, in (2), the dynamic relationship between the behaviour of the agent and the changes in the environment is modelled. The behaviour of agent A can be represented by its action function $a_{A}(t) \forall t$, defined as a correspondence between one moment in time $t$ and the action selected by the agent, 


$$
\text { Agent } A=\left\{a_{A}(t)\right\}_{t \in T \subseteq N}
$$

From the definition of the action function $a_{A}(t)$ a new relationship that collects the idea of an agent's action plan (3) can be defined,

$$
p_{A}: \underset{\left(t, a_{A}(t)\right)}{\operatorname{Tx} A} \rightarrow \underset{p_{A}(t)}{A}
$$

in the following way,

$$
p_{A}\left(t_{n}\right)=\sum_{i=1}^{n} a_{i A}\left(t_{i}-t_{i-1}\right)
$$

Given the dynamic character that we want to print onto our agent, the continuous extension of the previous expression (4) is proposed as a definition of the agent plan, in other words (5) -

$$
p_{A}\left(t_{n}\right)=\int_{t_{0}}^{t_{n}} a_{A}(t) d t
$$

The variation of the agent plan $p_{A}(t)$ will be provoked essentially by: the changes that occur in the environment and that force the initial plan to be modified, and the knowledge from the success and failure of the plans that were used in the past, and which are favoured or punished via learning. The planning is carried out through a neural network based on the Kohonen network [9]. The neurons are organized in a two-layer unidirectional architecture. The learning method is presented as follows: (The equations are presented in the order in that they should be executed).

- $\quad$ To present the input vector $\mathrm{X}^{p}=\left(x_{1}^{p}, \ldots, x_{i}^{p}, \ldots, x_{N}^{p}\right)^{T}$ in the input layer.

- The weightings initially take random weightings in $(0,1)$.

- To calculate the intensity of the neurons of the output layer. The Euclidean distance:

$$
y_{k}=\sqrt{\sum_{i=1}^{N}\left(x_{i}-w_{k i}\right)^{2}}
$$

- To determine the winning neuron that will be that of smaller Euclidean distance.

- To upgrade the weights of the neurons that connect the input layer with the output neuron:

$$
w_{k i}(t+1)=w_{k i}(t)+\eta(t) g(k, h, t)\left(x_{i}(t)-w_{k i}(t)\right)
$$

Where:

$$
g(k, h, t)=e^{\frac{-|k-h|^{2}}{2 R(t)^{2}}}
$$


(Gaussian function), therefore the formula is the following:

$$
w_{k i}(t+1)=w_{k i}(t)+\eta(t) e^{\frac{-|k-h|^{2}}{2 R(t)^{2}}}\left(x_{i}(t)-w_{k i}(t)\right)
$$

Where $w_{k i}$ is the weight of the connection between the input neuron $i$ and the output neuron $k ; t$ is the iterations; $\eta$ is the learning rate; $h$ is the position of the winning neuron; $k$ is the neuron of the output layer; and $i$ is the neuron of the input layer. In $k-h$ a distance is calculated between the neurons. The Euclidean distance has been used.

\section{Defining Self-Organising Neural Network in a novel Way: FYDPS Neural Network}

The basic Kohonen network [10] cannot be used to resolve our problem since it attempts to minimise distances without taking into account any other type of restriction, such as time limits. In the present study a planner is described that is based on Kohonen networks but with a number of improvements (FYDPS Neural Network) [13] that allow us to reach a solution far more rapidly. Furthermore, once a solution has been reached, it is re-modified in order to take restrictions into account.

\subsection{Objective 1: Reach a Solution more Rapidly}

As such, for this modification of the basic algorithm (FYDPS), we are aiming to make the solution search more agile and in order to achieve this, the basic vicinity function used in the Kohonen network is modified and the number of neurones in the output layer corresponds to the places that the subject wishes to visit. The topology of the neural network being considered is described below. The input layer is formed by two neurons, each one of those receives one of the coordinates of the patient's room presented as input. A vector of neurons is used of size the same as the number of places to visit by nurses of the problem in the output layer, as in [9] [11]. The number of neurons in the output layer isn't modified. Let $x_{i} \equiv\left(x_{i 1}, x_{i 2}\right) \quad i=1, \cdots . N$ the coordinates from the patient's room $i$ and $n_{i} \equiv\left(n_{i 1}, n_{i 2}\right) \quad i=1, \cdots, N$ the coordinates the neurons $i$ in $\mathfrak{R}^{2}$. $N$ rooms will be visited by the fixed nurse. Then there will be: Two neurons in the input layer and $N$ neurons in the output layer. It will be considered a vicinity function decreasing with the number of iterations.

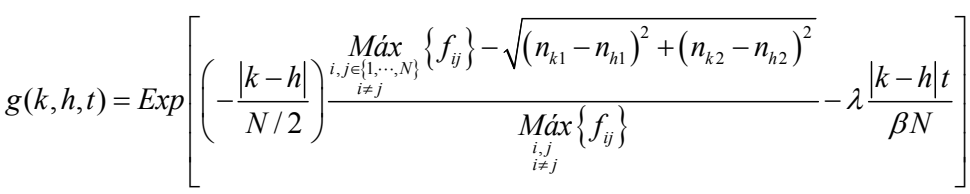


$\lambda$ and $\beta$ are determined empirically, their respective values are: 5 and $50 . t$ is the current iteration. $\operatorname{Exp}[x]=e^{x} . N$ is the number of the rooms that are visited by a fixed nurse and $f_{i j}$ is the distance given by the Floyd Algorithm [14].

The radius of final vicinity should be similar to 0 so that the winner is only upgraded. Iteratively the group of rooms will be presented, so that the weights of the neurons approach the coordinates of the rooms. When concluding the process, there will be a neuron associated to each room. To determine the route to follow, we will leave the room associated to the neuron $i$ to the associated to $i+1$, for $i=1,2, \ldots, N$, passing the whole vector of neurons. To close the road, the last tract will be given by the route that joins the patient's room associated to the neuron $N$ with the associated to the neuron 1 . The distance of the road will be given by the sum of the distances between the successive couples of rooms of the road. The learning rate is a decreasing function:

$$
\eta(t)=\operatorname{Exp}\left[-\sqrt[4]{\frac{t}{\beta N}}\right]
$$

The function of activation of the neurons is the identity. When the system stops, the route to continue will be given by the weights of the neurons that will be very closer to the coordinates of the rooms. To know which the following room in the journey, we pass to the following element of the vector of neurons. The neurons are stored in a vector that contains the weights of each one in the current instant. So that the vector defines a ring, the neuron $n_{1}$ is the following to the $n_{N}$ considered.

With a big radius of vicinity, in the first iterations of the algorithm the victory of a neuron affects great part of the map, so that a global self-organization takes place. If the radius decreases, the effect of a victory affects every time a part smaller in the map, so that the criterion to stop the learning of the network is that the distance among rooms cannot be optimized more. The initial number of total iterations is of $T_{1}=\beta N$ (first phase). When $t=\beta N$, all the couples of possible neurons are exchanged (exchanging their weights) in the obtained ring of neurons, if the distance is optimized then the learning isn't finished.

In general, in the phase $Z$, the total number of interactions to carry is:

$$
T_{Z}=T_{Z-1}-\frac{T_{Z-1}}{Z}
$$

The aim of these phases is to eliminate the crossings. Concluded the iterations of each phase is proven if the distance is already optimized, in such a way that in the phase that stops the learning, the distance is minimum. The primary objective - to achieve artificial neural networks that are faster than basic Kohonen networks, applied to the problems that the basic networks resolve - has been achieved. In the apparatus below the necessary modifications are introduced into the algorithm so that the network can take restrictions into account, and therefore be able to resolve other problems that cannot be resolved by basic Kohonen networks. 


\subsection{Objective 2: Taking Time Restrictions into Account}

Instead of using the Euclidean distance, a different distance is used that we call "temporal distance". Without loosing any generality it can be supposed that a distance unit is equivalent to a time unit.

$$
d t_{i j} \equiv d t\left(x_{i}, x_{j}\right) \square \operatorname{Máx}\left\{f_{i j}+t_{i}, b_{j}\right\}
$$

Where $t_{i}$ is the time hended to get to room " $i$ " from the previous room plus the time taken on tasks to be carried out within room " $i$ " (in other words, the service time in " $i$ ") and $b_{j}$ is the time limit for carrying out the tasks in room " $j$ ". In this way, the vicinity function of the network modified from the FYDPS network is:

$$
g(k, h, t)=\operatorname{Exp}\left[\left(-\frac{|k-h|}{N / 2}\right) \frac{\underset{\substack{i, j \in\{1, \cdots, \cdots, N\} \\ i \neq j}}{\operatorname{aax}}\left\{d_{i j}^{*}\right\}-\sqrt{\left(n_{k 1}-n_{h 1}\right)^{2}+\left(n_{k 2}-n_{h 2}\right)^{2}}}{\operatorname{Má}_{\substack{i, j \\ i \neq j}}\left\{d_{i j}^{*}\right\}}-\lambda \frac{|k-h| t}{\beta N}\right]
$$

With:

$$
d_{i j}^{*} \equiv d^{*}\left(x_{i}, x_{j}\right) \square \frac{f_{i j}+t_{i}}{d t_{i j}}
$$

When the network obtains as a result the optimum plan $p^{*}$. If the plan $p^{*}$ is not interrupted, the agent will reach a desired state $e_{j} \equiv e^{*}$. In the learning phase, a weighting $w_{f}(p)$ is stored. With the updating of weighting $w_{f}\left(p^{*}\right)$, the planning cycle of the CBP motor is completed. Let's suppose that the agent has initiated a plan $p^{*}$ but at a moment $t>t_{0}$, the plan is interrupted due to a change in the environment. The solutions given by the neural network meet the conditions of the Bellman Principle of Optimality [1], in other words, each on of the plan's parts is partially optimum between the selected points. This guarantees that if $g_{0}$ is optimum route for interrupted $e_{0}$ in $t_{l}$, because $e_{0}$ changes to $e_{1}$, and $g_{l}$ optimum route to $e_{1}$ that is begun in the state where $g_{0}$ has been interrupted, it follows that: $g=g_{0}+g_{l}$ is optimum route to $e=e_{0}\left(t_{1}-t_{0}\right)+e_{1}\left(t_{2}-t_{1}\right)$.

\section{Case Study}

The Alzheimer ST Residence of Salamanca has been interested in improving the services offered to its patients and has collaborated in the development of a prototype [5], [6]. Figure 1 shows a diagram of the technology implemented in the first floor of the ST Residence of Salamanca. We selected 30 patients to test the system, so the hardware implemented at the Residence basically consisted of 42 RFID door readers, one on each door and elevator, 4 controllers, one at each exit, one in the first floor hall and another in the second floor hall, and 36 bracelets, one for each patient and the nurses. The ID door readers get the ID number from the bracelets and send the data to 
the controllers which send a notification to the Manager agent. To test the system 30 patient agents, 10 nurse agents, 2 doctor agents and 1 manager agent were instantiated. AGALZ agent is an autonomous planner agent which schedules the nurse's working day obtaining dynamic plans depending on the tasks needed for each assigned patient. More details can be seen in [5], [6].

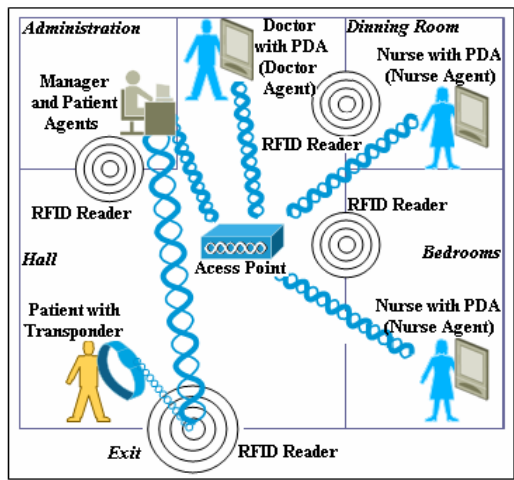

Fig. 1. ALZ-MAS wireless technology organization schema.

\section{Results and Conclusions}

The planning model proposed has been integrated within a previously Developed multiagent system [5], [6]. The tasks executed by nurses were divided in two categories, direct action tasks and indirect action tasks. AGALZ can act on the indirect action tasks. Averages of the time spent by nurses in the carrying out of the tasks for every patient were obtained, having into account that a task depends on the dependency level of a patient and the nurse skill. For the direct action tasks, the following times were obtained for each patient: 35' cleaning, 18' feeding, 8' oral medication, 30' parenteral medication, 25' posture change, 8' toileting, 60' exercise and $10^{\prime}$ others. We are especially interested on time spent on indirect action tasks; daily average times obtained for every kind of task before and after the implementation for each planner can be seen on Table 1.

Table 1. Time (minutes) spent on indirect tasks

\begin{tabular}{cccccc}
\hline & Monitoring & Reports & Visits & Other & TOTAL \\
\hline Before & 167 & 48 & 73 & 82 & 370 \\
Geodesic Planner & 105 & 40 & 45 & 60 & 250 \\
FYDPS Planner & 95 & 37 & 45 & 52 & 229 \\
\hline
\end{tabular}

The system facilitates the more flexible assignation of the working shifts at the residence; since the workers have reduced the time spent on routine tasks and can assign this time to extra activities. Their work is automatically monitored, as well as the patients' activities. The new planning mechanism schedules the tasks in a more efficient way. Table 2 shows a comparison an average time used by each kind of planner for generating a plan. 
Table 2. Planners execution time.

\begin{tabular}{cc}
\hline Planner & Execution Time (seconds) \\
\hline Geodesic Planner & 167 \\
FYDPS Planner & 95 \\
\hline
\end{tabular}

In the future, health care will require the use of new technologies that allow medical personnel to carry out their tasks more efficiently [3]. We have shown some potential of deliberative CBP-BDI agents in a distributed multi-agent system focused on health care. In addition, the use of RFID technology [15] on people provided a high level of interaction among users and patients through the system.

Acknowledgements. This work has been partially supported by the MCYT TIC200307369-C02-02 and the JCYL-2002-05 project SA104A05. Special thanks to Sokymat by the RFID technology provided and to Telefónica Móviles (Movistar) for the wireless devices donated.

\section{References}

1. Bellman R.E., Dynamic Programming. (Princeton University Press, Princeton, New Jersey, 1957).

2. Bratman, M.E.: Intentions, Plans and Practical Reason. Harvard University Press, Cambridge, M.A. (1987)

3. Camarinha-Matos L., and Afsarmanesh H., Design of a virtual community infrastructure for elderly care. PRO-VE'02. Sesimbra, Portugal. (2002).

4. Corchado J.M. and Laza R.: Constructing Deliberative Agents with Case-based Reasoning Technology, Int. Journal of Intelligent Systems. Vol 18, No. 12, pp. 1227-1241 (2003)

5. Corchado, J.M., Bajo, J., Tapia, D.I.: ALZ-MAS: Alzheimer's special care multi-agent system. Proceedings of the workshop on Health Care. ECAI'06. (2006)

6. Corchado J.M., Bajo J., de Paz Y. and Tapia D. I.: Intelligent Environment for Monitoring Alzheimer Patients, Agent Technology for Health Care. Decision Support Systems. Eslevier Science. DOI 10.1016/j.dss.2007.04.008. In Press (2007)

7. Cox M.T., Muñoz-Avila, H. and Bergmann R. Case-based planning. The Knowledge Engineering Review, Vol. 00:0, 1-4. c 2005, Cambridge University Press

8. Glez-Bedia M. and Corchado J. M.: A planning strategy based on variational calculus for deliberative agents. Computing and Information Systems Journal. Vol 10, No 1, 2002. ISBN: 1352-9404, pp. 2-14 (2002)

9. Jin H.D., Leung K.S., Wong M.L., Xu Z.B.: An Efficient Self-Organizing Map Designed by Genetic Algorithms for the Traveling Salesman Problem. IEEE Transactions on Systems, Man, and Cybernetics Part B: Cybernetics, vol. 33, no 6. (2003). pp 877-888.

10. Kohonen T.: Self-organization and associative memory, Springer Verlag (1984).

11. Leung K.S., Jin H.D., Xu Z.B.: An expanding Self-organizing Neural Network for the Traveling Salesman Problem. Neurocomputing, vol. 62. (2004). pp 267-292.

12. Marrow P. Nature-Inspired computing technology and applications. BT Technology Journal, vol. 18, $n^{\circ}$ 4. (2000)

13. Martín Q., De Paz J.F., De Paz Y., Pérez E., Solving TSP with a modified kohonen network, European Journal of Operational Research, (2007).

14. Martín Q., Santos M.T., De Paz Y., Operations research: Resolute problems and exercises, Pearson, (2005) 189-190.

15. Sokymat. Sokymat. http://www.sokymat.com. (2006) 